\title{
Delayed Spontaneous Pneumothorax in the COVID-19 Patient in the Highlander
}

\author{
Sadaf Samrina ${ }^{1}$, Kanwal Nimra ${ }^{1}$, Sualeh Mohammad Asad ${ }^{1}$ and Iqbal \\ Ratnani $^{1,2 *}$ \\ ${ }^{1}$ Intensive Care Unit, Agha Khan Medical Centre Gilgit, Pakistan \\ ${ }^{2}$ Houston Methodist Hospital, Texas, USA \\ *Corresponding Author: Iqbal Ratnani, Intensive Care Unit, Agha Khan Medical \\ Centre Gilgit, Pakistan.
}

Received: May 19, 2021

Published: June 24, 2021

(C) All rights are reserved by Iqbal Ratnani., et al.

\begin{abstract}
Covid-19 is a pandemic caused by SARS-Cov-2 primarily affecting the respiratory tract. However, there is scarce evidence of the association of a delayed spontaneous pneumothorax in a spontaneously breathing patient with covid-19. This report describes a patient with covid-19 who developed spontaneous pneumothorax after 20 days during infection without any underlying pulmonary predisposing factors. Pneumothorax in these patients if treated promptly has no bearing on clinical outcome.
\end{abstract}

Keywords: COVID-19; SARS-CoV-2; Spontaneous Pneumothorax; Coronavirus; Pneumothorax

\section{Introduction}

Coronavirus disease 2019 (COVID-19) infection is a global infection that affects many countries, including Pakistan. This infection has a broad spectrum of presentations that can range from asymptomatic disease to fatal acute respiratory distress syndrome.

Spontaneous pneumothorax is a rare complication of COVID-19. Most of the reported cases of pneumothorax associated with COVID-19 have traditional risk factors or underlying predisposing lung disease [1-11]. This case report describes the clinical course with covid-19 infection in a spontaneously breathing patient with no major underlying pulmonary risk factors for delayed pneumothorax. Pneumothorax in these patients if treated promptly has no bearing on clinical outcome.

\section{Case Presentation}

An 85-year-old gentleman with known hypertensive, ischemic heart disease (dual vessel coronary artery disease PCI done 2016) presented with complaints of fever, dry cough and dyspnoea for three days. He was a non-smoker. On examination at presentation, his $\mathrm{BP}$ was $130 / 70 \mathrm{mmHg}$, pulse rate of $92 / \mathrm{min}, \mathrm{SPO}_{2}$ was
$80 \%$ at room air, $95 \%$ with face mask at 5 liters/min. He was afebrile. On chest examination, there was bilateral crepitation at the lower chest. Other system examinations were unremarkable. Laboratory investigations showed a Haemoglobin of $12.5 \mathrm{mg} / \mathrm{dl}$, platelet count of 388,000 , total leukocyte count of $12.5 \times 10^{9}$ with neutrophils $93 \%$, and lymphocytes of $4 \%$. C-Reactive Protein was elevated. Chest X-ray showed bilaterally scattered infiltrates more prominent in lower zones (Figure 1). His nasopharyngeal COVID PCR was positive. Liver function test and renal function were unremarkable. He received COVID 19 treatment with Remdesivir $200 \mathrm{mg}$ loading dose, followed with $100 \mathrm{mg}$ daily for the next four days, Dexamethasone $6 \mathrm{mg}$ intravenous two times daily, injection Enoxaparin $40 \mathrm{mg}$ subcutaneous two times daily, injection Omeprazole $40 \mathrm{mg}$ daily.

His oxygen demand progressively increased during his hospital stay and was shifted from face mask to non-rebreathable mask up to 15 liters $\mathrm{FIO}_{2}$. On the $20^{\text {th }}$ day of admission, his dyspnoea worsened. On examination, there was decreased air entry on the right side. Chest X-ray was repeated, which showed right-sided pneumothorax involving more than $50 \%$ of right hemithorax (Figure 2). 


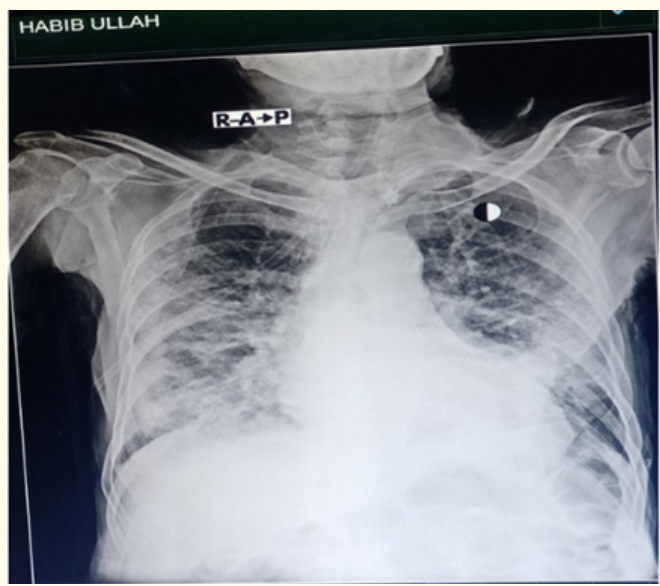

Figure 1: Chest X-ray AP view on presentation, showing bilateral patchy infiltrate, worse in the right lower lobe segments.

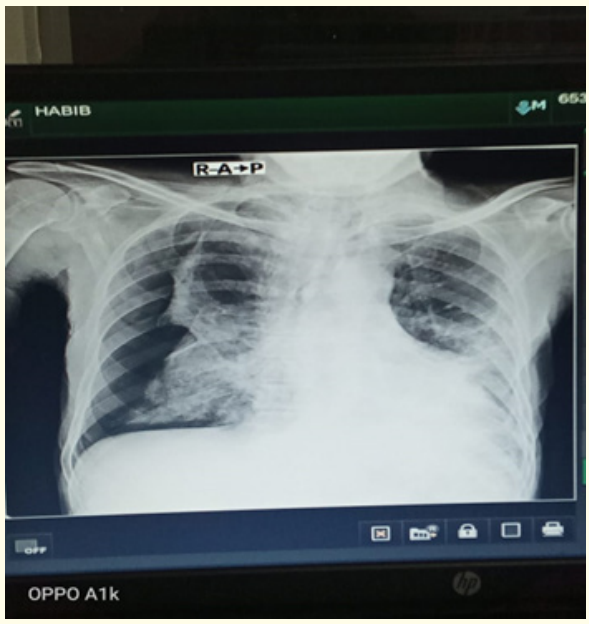

Figure 2: Chest X-ray showing right-sided pneumothorax (before tube thoracostomy).

Tube thoracostomy on right $5^{\text {th }}$ ICS was done under aseptic measures after informed consent. Repeat chest x-ray after tube thoracostomy showed resolution of pneumothorax (Figure 3). His arterial blood gases on nonrebreathable mask (NRM) showed hypoxemia with $\mathrm{PO}_{2}$ of $51.3, \mathrm{PCO}_{2}$ of $36.4, \mathrm{SPO}_{2}$ of $87 \%$, $\mathrm{PH} 7.45, \mathrm{HCO}_{3} 25.9$, for which he was started on non-invasive therapy with CPAP of $8 \mathrm{~cm}$ $\mathrm{H}_{2} \mathrm{O}$ overnight. Repeat arterial blood gases that morning showed $\mathrm{PH} 7.41, \mathrm{PO}_{2} 56.6, \mathrm{PCO}_{2} 36.0$ and $\mathrm{SPO}_{2} 92.2 \%, \mathrm{HCO}_{3}$ 30.6. Repeat chest x-ray after overnight CPAP showed worsening of pneumothorax and subcutaneous emphysema on the right side. The patient was not tolerating CPAP. His pneumothorax size increased with the positive airway pressure; therefore, he was switched to NRM with up to 15 liters, and he was maintaining $\mathrm{SPO}_{2}$ of more than $92 \%$. His chest tube remained for nine days. During this time, his oxygen requirement improved. On the $9^{\text {th }}$ day, the chest tube was removed, and he was maintaining SPO2 with 2 liters on a nasal cannula (Figure 4). He was mobilized fully and was discharged home on oneliter oxygen via nasal cannula with outpatient follow-up.

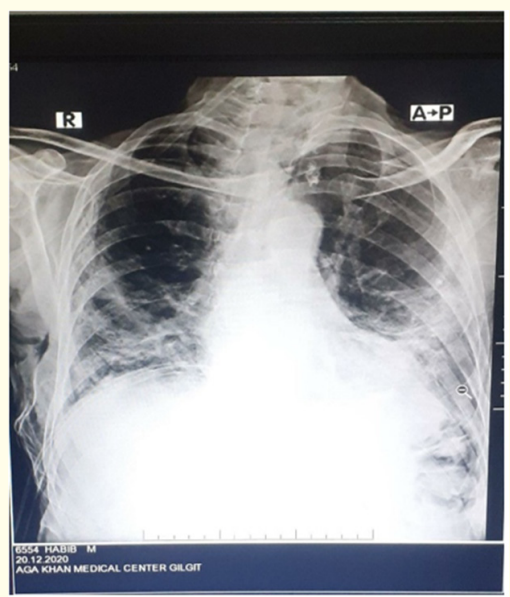

Figure 3: Chest X-ray post right-sided tube thoracostomy.

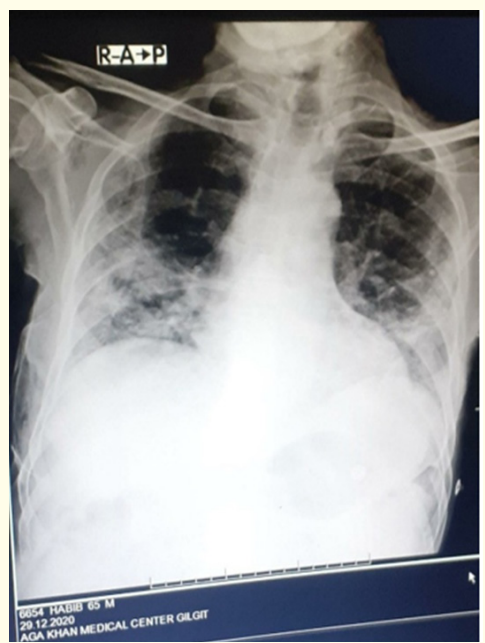

Figure 4: Chest X-ray after removal of chest tube. 


\section{Discussion}

Coronavirus disease 2019 infection is a severe and pandemic global infection spread in many countries, including Pakistan. It affects mainly the lung due to its access smoothly to cells through the angiotensin-converting enzyme. These two receptors are present commonly in type II alveolar cells in the lung [3] Pneumothorax can be developed with primary or secondary spontaneous causes. It occurs mainly in patients with a history of smoking, COPD, pneumonia, or inpatient mechanical ventilation with pre-existing lung disease. In this case, spontaneous pneumothorax was developed after 20 days of admission during the hospital stay. The patient requirement of $\mathrm{fio}_{2}$ was increasing gradually with increasing density of bilateral lung infiltrates since hospital admission. Weiyi., et al. had a 62-year-old man who developed of pneumothorax after about 20 days of admission for COVID-19 infection [5]. Luke flower., et al. study in which a 36-year-old man was diagnosed with tension pneumothorax of 4-hour duration following a 3-week history of cough, fevers, and dyspnoea with positive PCR for COVID-19 infection [7]. Fahad., et al. described a case report in which a fiftysix-year-old male patient with covid-19 infection developed rightsided pneumothorax on 21-day admission [10]. Burcin., et al. and Suphi., et al. case reports, both patients were present with spontaneous pneumothorax in addition to other features of COVID-19 infection initially $[3,4]$.

Here, there are no identified risk factors for developing a pneumothorax. Same as with Sharma., et al. case of severe pneumothorax and pneumoperitoneum in a non-ventilated patient like other reported cases $[4,5,10,11]$.

\section{Conclusion}

Coronavirus disease 2019 infection may be complicated with spontaneous pneumothorax after a period of initial symptoms (delayed pneumothorax). Pneumothorax is an important differential for acute deterioration of covid-19 besides pulmonary embolism later in the acute phase. Positive airway pressure may worsen pneumothorax, which clinicians need to be on lookout for. Pneumothorax in these patients if treated promptly has no bearing on clinical outcome.

\section{Bibliography}

1. Lyu R and Li X. "Diagnosis and treatment of severe COVID-19 complicated with spontaneous pneumothorax: a case report". Advanced Ultrasound in Diagnosis and Therapy 4 (2020): 142146.
2. Rohailla S., et al. "SARS-CoV-2 infection associated with spontaneous pneumothorax". CMAJ 192 (2020): E510.

3. Ucpinar BA., et al. "Spontaneous pneumothorax and subcutaneous emphysema in COVID-19 patient: case report". Journal of Infectious Public Health 13 (2020): 887-889.

4. Aydın S., et al. "A case of spontaneous pneumothorax in COVID-19 pneumonia". Journal of Surgical Research 3 (2020): 96101.

5. Wang W., et al. "COVID-19 with spontaneous pneumothorax, pneumomediastinum and subcutaneous emphysema". Journal of Travel Medicine 27 (2020).

6. Poggiali E., et al. "COVID-19, chronic obstructive pulmonary disease and pneumothorax: afrightening triad". European Journal of Case Reports in Internal Medicine 7.7 (2020): 001742.

7. Flower L., et al. "Tension pneumothorax in a patient with COVID-19”. BMJ Case Report 13 (2020): e235861.

8. Sun R., et al. "Mediastinalemphysema, giantbulla, and pneumothorax developed during the course of COVID-19 pneumonia". Korean Journal of Radiology 21 (2020): 541-544.

9. Wang J., et al. "Spontaneous pneumomediastinum: a probable unusual complication of coronavirus disease 2019 (COVID-19) pneumonia". Korean Journal of Radiology 21 (2020): 627-628.

10. Fahad AM., et al. "Spontaneous pneumothorax as a complication in COVID-19 male patient: A case report". Clinical Case Report 8 (2020): 3115-3118.

11. Sharma M., et al. "An intriguing case of pneumoperitoneumin an patient with COVID-19: Do all pneumoperitoneum cases need surgery?" Cureus 12.12 (2020): e12279.

\section{Volume 5 Issue 7 July 2021}

(C) All rights are reserved by Iqbal Ratnani., et al. 